\title{
Full information acquisition and analysis of reflection high energy electron diffraction data for epitaxial growth processes
}

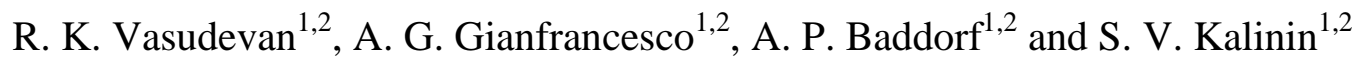 \\ 1. Institute for Functional Imaging of Materials, Oak Ridge National Laboratory, Oak Ridge TN 37831 \\ USA \\ 2. Center for Nanophase Materials Sciences, Oak Ridge National Laboratory, Oak Ridge TN 37831 USA
}

Reflection high energy electron diffraction (RHEED) has become ubiquitous in growth chambers worldwide, and has been used since the 1980s for in-situ characterization of thin films grown by both molecular beam epitaxy as well as pulsed laser deposition (PLD). The technique is essentially a surface electron diffraction technique where a beam of electrons is directed towards the substrate at small $(<\sim 1-$ $3^{\circ}$ ) grazing angles, resulting in a diffraction pattern that can provide information on the quality of the surface (roughness), the surface reconstructions, transitions between different growth modes, rate of growth of layers, etc. Given the non-ideal geometry of the diffraction as well as the dynamic nature of the scattering, most theoretical approaches to study the evolution of RHEED diffraction as the growth proceeds are highly involved, and as such application of RHEED is typically qualitative (or at most semi-quantitative). Much remains poorly understood about the time-evolution of RHEED diffraction patterns, and careful data analysis of the RHEED image sequences could open pathways towards both elucidation of the underlying physics, as well as control over the film growth process.

Here, we present a method for full information acquisition and analysis in RHEED [1], and apply it to two test cases where we show the ability to determine onsets of certain growth transitions. We study two commonly studied systems - PLD growth of a hole-doped manganite, $\mathrm{La}_{5 / 8} \mathrm{Ca}_{3 / 8} \mathrm{MnO}_{3}$ (LCMO), as well as $\mathrm{LaMnO}_{3}$ (LMO), both on (001) etched $\mathrm{SrTiO}_{3}$ substrates. The RHEED image patterns before and after the depositions are shown, along with the respective atomic force microscopy (AFM) topography images of the films in Fig. 1(a,b). The intensity evolution of the specular spot for both depositions is shown in Fig. 1(c). The LMO film was grown at $650{ }^{\circ} \mathrm{C}$ at $1 \mathrm{mTorr}_{2}$, while the LCMO film was grown $725^{\circ} \mathrm{C}$ at $10 \mathrm{mT}$ orr $\mathrm{O}_{2}$.

The RHEED images indicate that the LMO film is relatively smooth and flat, as confirmed by the topography image. However, the LCMO film displays clear 3D spots (additional diffraction spots above the specular reflection), and this further confirmed through the AFM topography image in Fig. 1b. To analyze this growth transition in more detail, we subjected the RHEED image sequences to principal component analysis (PCA), with the results for LCMO growth shown in Fig. 1(d,e). Analysis of the images reveals that the reflections associated with the 3D growth begin to appear at $t=180 \mathrm{~s}$. Accordingly, there are substantial changes in the time-dependent eigenvalues plotted in Fig. 1(d) around this time. More interestingly, in some components (e.g., the $5^{\text {th }}$ eigenvector) some intensity fluctuations can be observed just before the onset of the 3D growth. Whilst this may not be directly linked with the $3 \mathrm{D}$ growth, it warrants further investigation. From a physical standpoint, 3D growth regimes can be seen to occur when interatomic interactions are larger than between adatom and substrate [2], and when barriers to adatom diffusion are large (e.g. at island edges), leading to vertical growth of islands and thus 3D growth modes. These interactions are expected to be dependent on the substrate termination, the strain, the partial pressure of $\mathrm{O}_{2}$, and many other factors [3]. It can be expected that the growth of 3D islands would be preceded by an increase in the number of islands, which should reduce the intensity of 
the specular reflection as a result of increasing scattering from island edges. Notably, this is what is observed from the PCA results. These methods promise to provide greater insight into growth dynamics, and further advanced analysis should focus on establishing the link between the intensities of reflections and the onset of growth transitions, towards in-operando control over surface structures.

\section{References:}

[1] RK Vasudevan, A Tselev, AP Baddorf and SV Kalinin, ACS Nano 26(2015), p. 10899

[2] MSJ Marshall, MR Castell, Chemical Society Reviews 43 (2014), p. 2226

[3] A Tselev, RK Vasudevan, AG Gianfrancesco, Q Liang, P Ganesh, TL Meyer, HN Lee, MD

Biegalski, AP Baddorf and SV Kalinin, ACS Nano 9 (2015), p. 4316

[4] This research was sponsored by the Division of Materials Sciences and Engineering, BES, DOE (RKV, SVK). Research was conducted at the Center for Nanophase Materials Sciences, which also provided support (APB) and is a DOE Office of Science User Facility

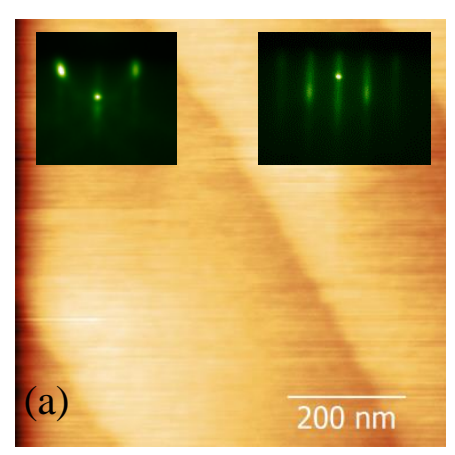

(d)
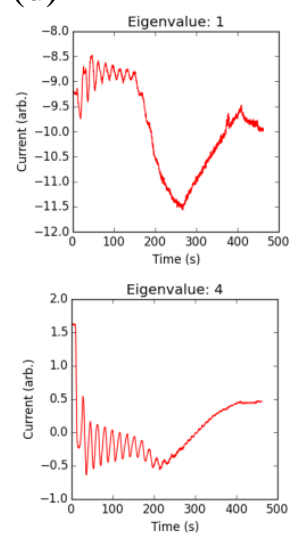

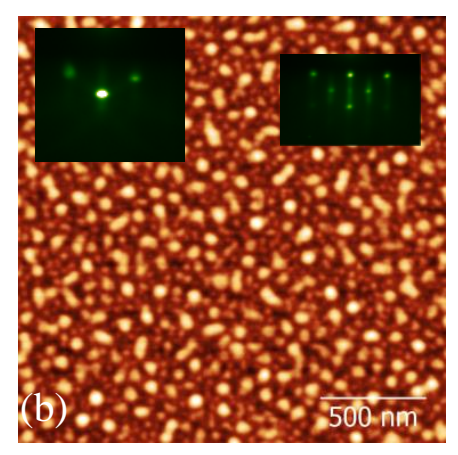

(e)
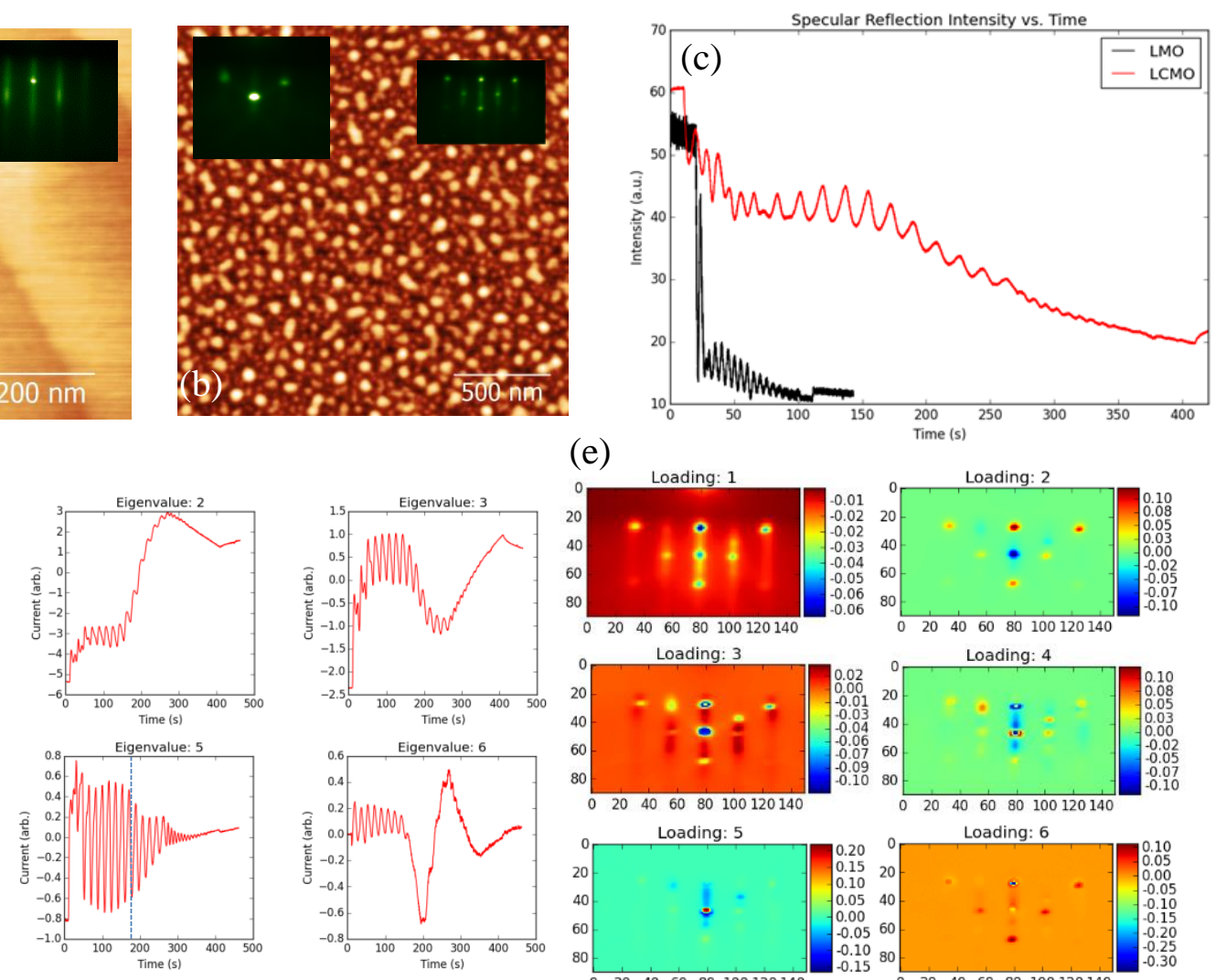

Loading: 1
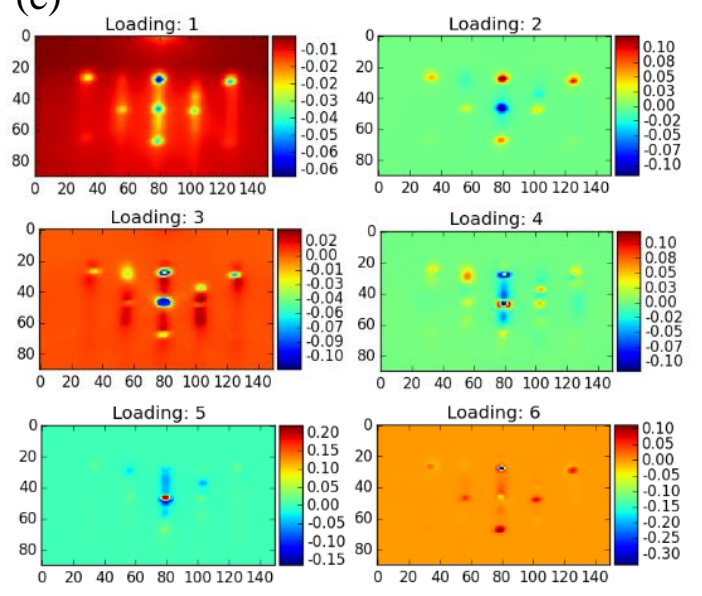

Figure 1. (a) AFM Topography of LMO film. (b) AFM topography of LCMO film along with the RHEED images before (inset, left) and after (inset, right) for both films. (c) Time evolution of the intensity of the specular spot for both depositions. (d,e) PCA of RHEED image sequence from LCMO film deposition. (d) PCA Eigenvalues and (e) Eigenvectors. The time at which the 3D growth diffraction spots appear is indicated by the dashed line in eigenvalue 5 . 